\title{
VISCOSITY APPROXIMATION METHOD FOR MODIFIED SPLIT GENERALIZED EQUILIBRIUM AND FIXED POINT PROBLEMS
}

\author{
HAMMED ANUOLUWAPO ABASS, CHINEDU IZUCHUKWU, \\ AND OLUWATOSIN TEMITOPE MEWOMO
}

\begin{abstract}
We introduce a viscosity iterative algorithm for approximating a common solution of a modified split generalized equilibrium problem and a fixed point problem for a quasi-pseudocontractive mapping which also solves some variational inequality problems in real Hilbert spaces. The proposed iterative algorithm is constructed in such a way that it does not require the prior knowledge of the operator norm. Furthermore, we prove a strong convergence theorem for approximating the common solution of the aforementioned problems. Finally, we give a numerical example of our main theorem. Our result complements and extends some related works in the literature.
\end{abstract}

\section{INTRODUCTION}

Let $C$ and $Q$ be nonempty closed convex subsets of real Hilbert spaces $H_{1}$ and $H_{2}$ respectively. The Split Feasibility Problem (SFP), first introduced in [7] by Censor and Elfving, requires finding a point in a nonempty closed convex subset in one space such that its image under a bounded linear operator is in another nonempty closed convex subset in the image space. That is, find $x^{*} \in C$ such that

$$
A x^{*} \in Q,
$$

where $A: H_{1} \rightarrow H_{2}$ is a bounded linear operator. The SFP arises in many fields in the real world, such as signal processing, image reconstruction, and intensitymodulated radiation therapy problems. For example, see [8, 9, 23] and the references therein. Many well-known iterative algorithms have been established for the SFP; for instance, Byrne [5] proposed the CQ algorithm to study the SFP; Qu and Xiu [20] considered a modified CQ algorithm to study the SFP; and Xu [26]

2010 Mathematics Subject Classification. Primary 47H06, 47H09, 47J05, 47J25.

Key words and phrases. Modified split generalized equilibrium problem; Variational inequality problem; Quasi-pseudocontractive mappings; Viscosity iterative scheme; Fixed point problem.

The first author acknowledges with thanks the bursary and financial support from Department of Science and Technology and National Research Foundation, Republic of South Africa Center of Excellence in Mathematical and Statistical Sciences (DST-NRF COE-MaSS) Doctoral Bursary. Opinions expressed and conclusions arrived are those of the authors and are not necessarily to be attributed to the CoE-MaSS. 
introduced a regularized algorithm for studying the SFP and proved a strong convergence result.

The introduction of the SFP to fixed point theory has also yielded some optimization problems such as the split equilibrium problem, the split variational inequality problem, the split inclusion poblem, among others.

Let $A: C \rightarrow H$ be a mapping. The Variational Inequality Problem (VIP) is to find $u \in C$ such that

$$
\langle A u, v-u\rangle \geq 0
$$

for all $v \in C$. The solution set of 1.1 is denoted by $\operatorname{VIP}(C, A)$. The VIP has emerged as a fascinating branch of mathematical and engineering sciences, with a wide range of applications in industry, finance, economics, ecology, and pure and applied sciences; see, for instance, [11, 17, 25.

Another optimization problem which includes the VIP is the Equilibrium Problem (EP), first introduced and studied by Blum and Oettli 44; see also 24. Many problems in physics, optimization, and economics can be reduced to finding the solution of EP, which is defined as follows: find $x \in C$ such that

$$
F(x, y) \geq 0,
$$

for all $y \in C$, where $F: C \times C \rightarrow \mathbb{R}$ is a bifunction. We denote by EP $(1.2)$ the solution set of 1.2 .

Let $F: C \times C \rightarrow \mathbb{R}$ be a bifunction and $f: H \rightarrow H$ a mapping. The Generalized Equilibrium Problem (GEP) is to find $x \in C$ such that

$$
F(x, y)+\langle f(x), y-x\rangle \geq 0,
$$

for all $y \in C$. We denote by $\operatorname{EP}(F, f)$ the solution set of 1.3 .

Remark 1.1. If $F \equiv 0$, the GEP 1.3 reduces to VIP 1.1 , and when $f \equiv 0$, the GEP 1.3 reduces to EP 1.2.

In 2013, Kazmi and Rizvi [18 introduced and studied the following Split Equilibrium Problem (SEP), which is to find $x^{*} \in C$ such that

$$
F_{1}\left(x^{*}, x\right) \geq 0, \quad \forall x \in C,
$$

and such that

$$
y^{*}=A x^{*} \in Q \quad \text { solves } \quad F_{2}\left(y^{*}, y\right) \geq 0, \quad \forall y \in Q,
$$

where $F_{1}: C \times C \rightarrow \mathbb{R}$ and $F_{2}: Q \times Q \rightarrow \mathbb{R}$ are nonlinear bifunctions.

The SEP 1.4 - 1.5 reduces to EP 1.2 , if $H_{1} \equiv H_{2}, F_{1} \equiv F_{2}, A \equiv I$, and $C=Q$.

The Split Variational Inequality Problem (SVIP) was introduced and studied by Censor et al. [10], who defined the problem as follows: find $x^{*} \in C$ such that

$$
\left\langle f_{1}\left(x^{*}\right), x-x^{*}\right\rangle \geq 0, \quad \forall x \in C,
$$

and such that

$$
y^{*}=A x^{*} \in Q \quad \text { solves }\left\langle f_{2}\left(y^{*}\right), y-y^{*}\right\rangle \geq 0, \quad \forall y \in Q,
$$


where $f_{1}: C \rightarrow H_{1}$ and $f_{2}: Q \rightarrow H_{2}$ are nonlinear mappings. The SVIP has already been used in practice as a model in intensity-modulated radiation therapy (IMRT) treatment planning and the modelling of many inverse problems arising from phase retrieval and other real-world problems such as data compression or sensor networks in computerized tomography; see for example [14.

Very recently, Cheawchan and Kangtunyakarn [13] introduced the Modified Split Generalized Equilibrium Problem (MSGEP), which is to find $x^{*} \in C$ such that

$$
F_{1}\left(x^{*}, x\right)+\left\langle f_{1}\left(x^{*}\right), x-x^{*}\right\rangle \geq 0, \quad \forall x \in C,
$$

and such that

$$
y^{*}=A x^{*} \in Q \quad \text { solves } \quad F_{2}\left(y^{*}, y\right)+\left\langle f_{2}\left(y^{*}\right), y-y^{*}\right\rangle \geq 0, \quad \forall y \in Q,
$$

where $F_{1}: C \times C \rightarrow \mathbb{R}$ and $F_{2}: Q \times Q \rightarrow \mathbb{R}$ are nonlinear bifunctions and $f_{1}: C \rightarrow H_{1}$ and $f_{2}: Q \rightarrow H_{2}$ are nonlinear mappings. We denote by $\Omega=\left\{x^{*} \in\right.$ $\left.\operatorname{EP}\left(F_{1}, f_{1}\right): A x^{*} \in \operatorname{EP}\left(F_{2}, f_{2}\right)\right\}$ the solution set of MSGEP (1.8)-(1.9).

The MSGEP generalizes the SEP (1.4)-(1.5) and the SVIP (1.6)-(1.7) in the following ways:

(i) if $f_{1} \equiv f_{2} \equiv 0$ in MSGEP $1.8-(1.9)$, then MSGEP $1.8-(1.9)$ reduces to SEP (1.4)- 1.5 ;

(ii) if $F_{1} \equiv F_{2} \equiv 0$ in MSGEP $(1.8)-(1.9)$, then MSGEP $(1.8)-(1.9)$ reduces to SVIP 1.6)-1.7.

For solving EP, we assume that the bifunction $F: C \times C \rightarrow \mathbb{R}$ satisfies the following conditions:

(A1) $F(x, x)=0$, for all $x \in C$;

(A2) $F$ is monotone, i.e. $F(x, y)+F(y, x) \leq 0, \forall x, y \in C$;

(A3) for each $x, y, z \in C$, $\lim \sup _{t \rightarrow 0} F(t z+(1-t) x, y) \leq F(x, y)$;

(A4) for each $x \in C, y \mapsto F(x, y)$ is convex and lower semi-continuous.

Let $r>0$ and $x \in H$. Then, there exists $z \in C$ such that

$$
F(z, y)+\frac{1}{r}\langle y-z, z-x\rangle \geq 0, \quad \forall y \in C .
$$

Let $C$ be a nonempty closed convex subset of a real Hilbert space $H$. For every point $x \in H$, there exists a unique nearest point in $C$, denoted by $P_{C} x$, such that

$$
\left\|x-P_{C}(x)\right\| \leq\|x-y\|, \quad \forall y \in C .
$$

$P_{C}$ is called the metric projection of $H$ onto $C$. It is well known that $P_{C}$ is a nonexpansive mapping of $H$ onto $C$ and satisfies

$$
\left\|P_{C}(x)-P_{C}(y)\right\| \leq\left\langle x-y, P_{C}(x)-P_{C}(y)\right\rangle .
$$

Moreover, $P_{C}(x)$ is characterized by the following properties:

$$
\left\langle x-P_{C}(x), y-P_{C}(x)\right\rangle \leq 0
$$

and

$$
\|x-y\|^{2} \geq\left\|x-P_{C}(x)\right\|^{2}+\left\|y-P_{C}(x)\right\|^{2}, \quad \forall x \in H, y \in C .
$$


For all $x, y \in H$, it is well known that every nonexpansive operator $T: H \rightarrow H$ satisfies the inequality

$$
\langle(x-T(x))-(y-T(y)), T(y)-T(x)\rangle \leq \frac{1}{2}\|(T(x)-x)-(T(y)-y)\|^{2},
$$

and therefore, we have that for all $x \in H$ and $y \in F(T)$.

$$
\langle x-T(x), y-T(x)\rangle \leq \frac{1}{2}\|T(x)-x\|^{2} .
$$

We now give some definitions that will be needed later.

Definition 1.2. Let $C$ be a nonempty closed convex subset of a real Hilbert space $H$. A point $p \in C$ is called a fixed point of a mapping $T$ if $T p=p$. We denote by $F(T)$ the set of all fixed points of $T$.

Definition 1.3. Let $C$ be a nonempty closed convex subset of a real Hilbert space $H$. We say that a nonlinear mapping $T: C \rightarrow C$ is

(i) a contraction, if there exists a constant $\phi \in(0,1)$ such that

$$
\|T x-T y\| \leq \phi\|x-y\|, \quad \forall x, y \in C ;
$$

(ii) nonexpansive, if

$$
\|T x-T y\| \leq\|x-y\|, \quad \forall x, y \in C ;
$$

(iii) firmly nonexpansive, if

$$
\|T x-T y\|^{2} \leq\|x-y\|^{2}-\|(I-T) x-(I-T) y\|^{2}, \quad \forall x, y \in C ;
$$

(iv) firmly quasi-nonexpansive, if $F(T) \neq \emptyset$ and

$$
\left\|T x-x^{*}\right\|^{2} \leq\left\|x-x^{*}\right\|^{2}-\|(I-T) x\|^{2}, \quad \forall x \in C \text { and } x^{*} \in F(T) ;
$$

(v) strictly pseudo-contractive if there exists $k \in(0,1]$ such that

$$
\|T x-T y\|^{2} \leq\|x-y\|^{2}+k\|(I-T) x-(I-T) y\|^{2}, \quad \forall x, y \in C ;
$$

(vi) demicontractive, if $F(T) \neq \emptyset$ and there exists $k \in[0,1)$ such that

$$
\left\|T x-x^{*}\right\|^{2} \leq\left\|x-x^{*}\right\|^{2}+k\|T x-x\|^{2}, \quad \forall x \in C \text { and } x^{*} \in F(T) .
$$

From the definitions stated above, we notice that the class of demicontractive mappings includes many nonlinear mappings, such as quasi-nonexpansive and strictly pseudo-contractive with nonempty fixed points sets, as special cases.

In 2015, Chang et al. [12] introduced a new type of nonlinear mapping called quasi-pseudo-contractive mapping, as follows:

Definition 1.4. An operator $T: C \rightarrow C$ is said to be quasi-pseudo-contractive if $F(T) \neq \emptyset$ and

$$
\left\|T x-x^{*}\right\|^{2} \leq\left\|x-x^{*}\right\|^{2}+\|T x-x\|^{2}, \quad \forall x \in C \text { and } x^{*} \in F(T) .
$$

It is obvious that this class of mappings contains the class of demicontractive mappings, see [12]. 
Definition 1.5. A bounded linear operator $D$ on $H$ is called strongly positive if there exists a constant $\beta>0$ such that

$$
\langle D x, x\rangle \geq \beta\|x\|^{2}, \quad \forall x \in C .
$$

Definition 1.6. Let $H$ be a real Hilbert space and $C$ be a nonempty closed convex subset of $H$. A mapping $T: C \rightarrow C$ is said to be demiclosed at 0 if for any bounded sequence $\left\{x_{n}\right\} \subset C$ such that $\left\{x_{n}\right\}$ converges weakly to $x$ and $\lim _{n \rightarrow \infty}\left\|x_{n}-T x_{n}\right\|=0$, we have that $T x=x$.

The viscosity iterative algorithm is one of the algorithms that have been used extensively by authors to approximate solutions of fixed point problems and optimization problems. The algorithm is constructed in such a way that it also solves some variational inequality problem (see [6, 21, 28, and the references therein). In 2017, Deepho et al. [16] considered the viscosity iterative algorithm to approximate a common element of the set of solutions of a split variational inclusion problem of a finite family of $k$-strictly pseudo-contractive nonself mappings. They proved a strong convergence result under suitable conditions, which also solves some variational inequality problem. The following iteration process was used to approximate the aforementioned problems:

$$
\left\{\begin{aligned}
u_{n} & =J_{\lambda}^{B_{1}}\left(x_{n}+\gamma A^{*}\left(J_{\lambda}^{B_{2}}\right) A x_{n}\right), \\
y_{n} & =\beta_{n} u_{n}+\left(1-\beta_{n}\right) \sum_{i=1}^{N} \eta_{i=1}^{n} T_{i} u_{n}, \\
x_{n+1} & =\alpha_{n} \tau g\left(x_{n}\right)+\left(I-\alpha_{n} D\right) y_{n}, \quad n \geq 1,
\end{aligned}\right.
$$

where $\alpha_{n}, \beta_{n} \in(0,1), \lambda>0, g$ is a contraction mapping with coefficient $\rho \in(0,1)$, $\sum_{i=1}^{N} \eta_{i=1}^{n}=1,\left\{T_{i}\right\}_{i=1}^{N}$ is a finite family of $k_{i}$-strictly pseudo-contraction mappings, and $J_{\lambda}^{B_{i}}(i=1,2)$ is the resolvent of the maximal monotone mappings.

In 2018, Abass et al. [1] introduced an iterative algorithm that does not require the prior knowledge of the operator norm to approximate the common solution of SEP and fixed point problem for an infinite family of quasi-nonexpansive multivalued mappings. Using their iterative algorithm, they prove a strong convergence result.

Very recently, Cheawchan and Kangtunyakarn [13] introduced a new iterative algorithm for finding a common element of the set of solutions of variational inequality problems and the set of solutions of MSGEP without assuming the demiclosedness condition. They proved the following theorem.

Theorem 1.7 ([13). Let $C$ and $Q$ be nonempty closed convex subsets of real Hilbert spaces $H_{1}$ and $H_{2}$ respectively. Let $A: H_{1} \rightarrow H_{2}$ be a bounded linear operator. Let $D_{1}, D_{2}: C \rightarrow H_{1}$ be $\alpha$-, $\beta$-inverse strongly monotone mappings respectively. Let $F_{1}: C \times C \rightarrow \mathbb{R}$ and $F_{2}: Q \times Q \rightarrow \mathbb{R}$ be bifunctions satisfying (A1)-(A4). Let $\left\{T_{i}\right\}_{i=1}^{\infty}$ be a finite family of quasi-nonexpansive mappings of $C$ into itself with $\cap_{i=1}^{N} F\left(T_{i}\right) \neq \emptyset$. Let $f_{1}: H_{1} \rightarrow H_{1}$ be a $\rho$-inverse strongly monotone mapping and $f_{2}: H_{2} \rightarrow H_{2}$ be a firmly nonexpansive mapping. Assume that 
$\Pi:=V I\left(C, D_{1}\right) \cap V I\left(C, D_{2}\right) \cap \cap_{i=1}^{N} F\left(T_{i}\right) \cap \Omega \neq \emptyset$. For $x_{1}, u \in C$, let $\left\{x_{n}\right\},\left\{u_{n}\right\}$, and $\left\{y_{n}\right\}$ be sequences generated by

$$
\left\{\begin{aligned}
u_{n} & =T_{r}^{F_{1}}\left(I-r f_{1}\right)\left(x_{n}+\gamma A^{*}\left(T_{s}^{F_{2}}\left(I-s f_{2}\right)-I\right) A x_{n}\right), \\
y_{n} & =P_{C}\left(I-d_{1} D_{1}\right)\left(a u_{n}+(I-a) P_{C}\left(I-d_{2} D_{2}\right) u_{n}\right), \\
x_{n+1} & =\alpha_{n} u+\beta_{n} x_{n}+\gamma_{n} P_{C}\left(I-\lambda_{n}\left(\sum_{i=1}^{N} k_{i}\left(I-T_{i}\right)\right)\right) y_{n}, \quad \forall n \in \mathbb{N},
\end{aligned}\right.
$$

where $d_{1} \in(0,2 \alpha), d_{2} \in(0,2 \beta), r \in(0,2 \rho), s \in(0,1), a \in[0,1], 0<k_{i}<1$, with $\sum_{i=1}^{N} k_{i}=1, \gamma \in\left(0, \frac{1}{L}\right), L$ is the spectral radius of the operator $A^{*} A$, and $A^{*}$ is the adjoint of $A$. Also, $\left\{\alpha_{n}\right\},\left\{\beta_{n}\right\},\left\{\gamma_{n}\right\}$ are sequences in $[0,1]$ with $\alpha_{n}+\beta_{n}+\gamma_{n}=1$ for all $n \in \mathbb{N}$. Suppose the following conditions hold:

(i) $\lim _{n \rightarrow \infty} \alpha_{n}=0$ and $\sum_{n=1}^{\infty} \alpha_{n}=\infty$;

(ii) $0<c \leq \beta_{n}, \gamma_{n} \leq d<1$ for some $c, d>0$ for all $n \geq 1$;

(iii) $\sum_{i=1}^{\infty} \lambda_{n}<\infty$ and $0<\lambda_{n}<1$;

(iv) $\sum_{n=1}^{\infty}\left|\alpha_{n+1}-\alpha_{n}\right|<\infty, \sum_{n=1}^{\infty}\left|\beta_{n+1}-\beta_{n}\right|<\infty$.

Then $\left\{x_{n}\right\}$ converges strongly to $z=P_{\Pi} u$.

Motivated by the works of Abass et al. [1, Deepho et al. [16], Cheawchan and Kangtunyakarn [13], we propose a viscosity iterative algorithm that does not require any knowledge of the spectral radii to approximate a common solution of MSGEP and fixed point problem for a quasi-pseudo-contractive mapping, which is also a solution of some variational inequality problem. We prove a strong convergence of the iterative scheme to a solution of the aforementioned problems in the framework of real Hilbert spaces. Furthermore, we give a numerical example of our main result.

\section{Preliminaries}

We state some known and useful results which will be needed in the proof of our main theorem. In what follows, we denote strong and weak convergence by " $\rightarrow$ " and " - ", respectively.

Lemma 2.1. Let $H$ be a real Hilbert space. Then for all $x, y \in H$ and $\alpha \in(0,1)$ we have

$$
\begin{aligned}
\|\alpha x+(1-\alpha) y\|^{2} & =\alpha\|x\|^{2}+(1-\alpha)\|y\|^{2}-\alpha(1-\alpha)\|x-y\|^{2} \\
\|x+y\|^{2} & \leq\|x\|^{2}+2\langle y, x+y\rangle ; \\
2\langle x, y\rangle & =\|x\|^{2}+\|y\|^{2}-\|x-y\|^{2}=\|x+y\|^{2}-\|x\|^{2}-\|y\|^{2} .
\end{aligned}
$$

Lemma 2.2 ([13]). Let $C$ and $Q$ be nonempty closed convex subsets of real Hilbert spaces $\mathrm{H}_{1}$ and $\mathrm{H}_{2}$ respectively. Let $F_{1}: C \times C \rightarrow \mathbb{R}$ and $F_{2}: Q \times Q \rightarrow \mathbb{R}$ be bifunctions satisfying (A1)-(A4). Let $f_{1}: H_{1} \rightarrow H_{1}$ be a $\rho$-inverse strongly monotone mapping and $f_{2}: H_{2} \rightarrow H_{2}$ be a firmly nonexpansive mapping. Then 
$T_{r}^{F_{1}}\left(I-r f_{1}\right)$ and $T_{s}^{F_{2}}\left(I-s f_{2}\right)$ are nonexpansive mappings, where $r \in(0,2 \rho)$, $s \in(0,1)$, and $T_{r}^{F_{1}}: H_{1}: C$ is defined by

$$
T_{r}^{F_{1}}(x)=\left\{z \in C: F_{1}(z, y)+\frac{1}{r}\langle y-z, z-x\rangle \geq 0, \forall y \in C\right\},
$$

for all $x \in H_{1}$ and $T_{s}^{F_{2}}: H_{2} \rightarrow Q$ is defined by

$$
T_{s}^{F_{2}}(\bar{x})=\left\{\bar{z} \in Q: F_{2}(\bar{z}, y)+\frac{1}{s}\langle y-\bar{z}, \bar{z}-\bar{x} \geq 0, \forall y \in Q\},\right.
$$

for all $\bar{x} \in \mathrm{H}_{2}$.

Recall that a Banach space $X$ is said to satisfy Opial's condition if for any sequence $\left\{x_{n}\right\}$ in $X$ which converges weakly to $x^{*}$,

$$
\limsup _{n \rightarrow \infty}\left\|x_{n}-x^{*}\right\|<\limsup _{n \rightarrow \infty}\left\|x_{n}-y\right\|, \quad \forall y \in X \text { with } y \neq x^{*} .
$$

Lemma $2.3([15])$. Let $C$ be a nonempty closed convex subset of a real Hilbert space $H$ and $F: C \times C \rightarrow \mathbb{R}$ be a bifunction satisfying (A1)-(A4). For $r>0$ and $x \in H$, define a mapping $T_{r}^{F}: H \rightarrow C$ as follows:

$$
T_{r}^{F} x=\left\{z \in C: F(z, y)+\frac{1}{r}\langle y-z, z-x\rangle \geq 0, \forall y \in C\right\},
$$

for all $x \in H$. Then the following hold:

(i) $T_{r}^{F}$ is nonempty and single-valued;

(ii) $T_{r}^{F}$ is firmly nonexpansive, that is, $\forall x, y \in H$,

$$
\left\|T_{r}^{F} x-T_{r}^{F} y\right\|^{2} \leq\left\langle T_{r}^{F} x-T_{r}^{F} y, x-y\right\rangle ;
$$

(iii) $F\left(T_{r}^{F}\right)=E P(F)$;

(iv) $E P(F)$ is closed and convex.

Lemma 2.4 ([19]). Assume that $D$ is a strongly positive bounded linear operator on a Hilbert space $H$ with a coefficient $\bar{\tau}>0$ and $0<\mu<\|D\|^{-1}$. Then $\|I-\mu D\| \leq$ $1-\mu \bar{\tau}$.

Lemma 2.5 ([12). Let $H$ be a real Hilbert space and $T: H \rightarrow H$ be an $L$ Lipschitzian mapping with $L \geq 1$. Denote $K:=(1-\xi) I+\xi T((1-\eta) I+\eta T)$ if $0<\xi<\eta<\frac{1}{1+\sqrt{1+L^{2}}}$. Then the following conclusions hold:

(1) $F(T)=F(T((1-\eta) I+\eta T))=F(K)$;

(2) if $T$ is demiclosed at 0 , then $K$ is also demiclosed at 0 ;

(3) in addition, if $T: H \rightarrow H$ is quasi-pseudocontractive, then the mapping $K$ is quasi-nonexpansive, that is,

$$
\left\|K x-u^{*}\right\| \leq\left\|x-u^{*}\right\|, \quad \forall x \in H \text { and } u^{*} \in F(T)=F(K) .
$$

Lemma 2.6 ([19]). Let $C$ be a nonempty closed convex subset of a real Hilbert space $H$. Assume that $f: C \rightarrow C$ is a contraction with coefficient $\mu \in(0,1)$ and $D$ is a strongly positive linear bounded operator with a coefficient $\bar{\sigma}>0$. Then, for $0<\sigma<\frac{\bar{\sigma}}{\mu}$,

$$
\langle x-y,(D-\sigma f) x-(D-\sigma f) y\rangle \geq(\bar{\sigma}-\sigma \mu)\|x-y\|^{2}, \quad x, y \in H .
$$


That is, $D-\sigma f$ is strongly monotone with coefficient $\bar{\sigma}-\sigma \mu$.

Lemma $2.7([26])$. Assume that $\left\{a_{n}\right\}$ is a sequence of nonnegative real numbers such that

$$
a_{n+1} \leq\left(1-\sigma_{n}\right) a_{n}+\sigma_{n} \delta_{n}, \quad n>0,
$$

where $\left\{\sigma_{n}\right\}$ is a sequence in $(0,1)$ and $\left\{\delta_{n}\right\}$ is a real sequence satisfying

(i) $\sum_{n=1}^{\infty} \sigma_{n}=\infty$,

(ii) $\limsup _{n \rightarrow \infty} \delta_{n} \leq 0$ or $\sum_{n=1}^{\infty}\left|\sigma_{n} \delta_{n}\right|<\infty$.

Then $\lim _{n \rightarrow \infty} a_{n}=0$.

\section{MAin Result}

Lemma 3.1. Let $C$ be a nonempty closed convex subset of a real Hilbert space $H$. Let $F: C \times C \rightarrow \mathbb{R}$ be a bifunction satisfying (A1)-(A4) and $f: H \rightarrow H$ be any nonlinear mapping. Then, for $0<r_{1} \leq r_{2}$, we have that

$$
\left\|T_{r_{1}}^{F}\left(I-r_{1} f\right) x-T_{r_{2}}^{F}\left(I-r_{2} f\right) x\right\| \leq\left\|x-T_{r_{2}}^{F}\left(I-r_{2} f\right) x\right\|, \quad \forall x \in H .
$$

Proof. Let $z=T_{r_{1}}^{F}\left(I-r_{1} f\right) x$ and $w=T_{r_{2}}^{F}\left(I-r_{2} f\right) x$. Then, from 2.1) we obtain

$$
F(z, w)+\frac{1}{r_{1}}\left\langle w-z, z-\left(I-r_{1} f\right) x\right\rangle \geq 0 .
$$

Similarly, we obtain

$$
F(w, z)+\frac{1}{r_{2}}\left\langle z-w, w-\left(I-r_{2} f\right) x\right\rangle \geq 0 .
$$

Adding the last two inequalities and using condition (A2) we obtain

$$
\left\langle x-z-r_{1} f x, z-w\right\rangle-\frac{r_{1}}{r_{2}}\left\langle x-w-r_{2} f x, z-w\right\rangle \geq 0 .
$$

That is,

$$
\left\langle\left(x-r_{1} f x-z\right)-\left(\frac{r_{1}}{r_{2}} x-r_{1} f x-\frac{r_{1}}{r_{2}} w\right), z-w\right\rangle \geq 0,
$$

which implies that

$$
\langle x-z, z-w\rangle \geq \frac{r_{1}}{r_{2}}\langle x-w, z-w\rangle .
$$

Thus, from Lemma 2.1 we obtain

$$
\|x-w\|^{2}-\|x-z\|^{2}-\|x-z\|^{2}-\|z-w\|^{2} \geq \frac{r_{1}}{r_{2}}\left(\|x-w\|^{2}+\|w-z\|^{2}-\|x-z\|^{2}\right) .
$$

Since $\frac{r_{1}}{r_{2}} \leq 1$, we get

$$
\left(1+\frac{r_{1}}{r_{2}}\right)\|z-w\|^{2} \leq\left(1-\frac{r_{1}}{r_{2}}\right)\|x-w\|^{2},
$$

which implies that

$$
\|z-w\|^{2} \leq\left(\frac{r_{2}-r_{1}}{r_{2}+r_{1}}\right)\|x-w\|^{2} \leq\|x-w\|^{2} .
$$


Lemma 3.2. Let $C$ and $Q$ be nonempty closed convex subsets of real Hilbert spaces $H_{1}$ and $H_{2}$ respectively. Let $A: H_{1} \rightarrow H_{2}$ be a bounded linear operator and $T: H_{1} \rightarrow H_{2}$ be an L-Lipschitzian and quasi-pseudocontractive mapping with $L \geq 1$. Let $F_{1}: C \times C \rightarrow \mathbb{R}, F_{2}: Q \times Q \rightarrow \mathbb{R}$ be bifunctions satisfying (A1)-(A4) and let $D$ be a strongly positive bounded linear operator on $H_{1}$ with coefficient $\bar{\tau}>0$. Let $f_{1}: H_{1} \rightarrow H_{1}$ be a $\rho_{1}$-inverse strongly monotone mapping and $f_{2}: H_{2} \rightarrow H_{2}$ be a $\rho_{2}$-inverse strongly monotone mapping. Assume that $\Gamma:=F(T) \cap \Omega \neq \emptyset$ and $g$ is a contraction mapping with coefficient $\mu \in(0,1)$. Let the sequences $\left\{u_{n}\right\},\left\{y_{n}\right\}$, and $\left\{x_{n}\right\}$ be generated for arbitrary $x_{1} \in H$ by

$$
\left\{\begin{aligned}
u_{n} & =T_{r_{n}}^{F_{1}}\left(I-r_{n} f_{1}\right)\left(x_{n}+\gamma_{n} A^{*}\left(T_{s_{n}}^{F_{2}}\left(I-s_{n} f_{2}\right)-I\right) A x_{n}\right), \\
y_{n} & =\alpha_{n} u_{n}+\left(1-\alpha_{n}\right)\left(\left(1-\xi_{n}\right) I+\xi_{n} T\left(1-\eta_{n}\right) I+\eta_{n} T\right) u_{n} \\
x_{n+1} & =\beta_{n} \tau g\left(x_{n}\right)+\left(I-\beta_{n} D\right) y_{n}, \quad n \geq 1
\end{aligned}\right.
$$

where $K:=\left(\left(1-\xi_{n}\right) I+\xi_{n} T\left(1-\eta_{n}\right) I+\eta_{n} T\right), 0<r \leq r_{n}<2 \rho_{1}, 0<s \leq s_{n}<2 \rho_{2}$, $\alpha_{n} \in(0,1)$, and the step size $\gamma_{n}$ is chosen in such a way that for some $\epsilon>0$,

$$
\gamma_{n} \in\left(\epsilon, \frac{\left\|\left(T_{s_{n}}^{F_{2}}\left(I-s_{n} f_{2}\right)-I\right) A x_{n}\right\|^{2}}{\left\|A^{*}\left(T_{s_{n}}^{F_{2}}\left(I-s_{n} f_{2}\right)-I\right) A x_{n}\right\|^{2}}-\epsilon\right),
$$

for all $T_{s_{n}}^{F_{2}}\left(I-s_{n} f_{2}\right) A x_{n} \neq A x_{n}$, and $\gamma_{n}=\gamma$ otherwise ( $\gamma$ being any nonnegative real number), with the sequence $\left\{\beta_{n}\right\}$ satisfying the following conditions:

(i) $\beta_{n} \in(0,1), \lim _{n \rightarrow \infty} \beta_{n}=0$, and $\sum_{n=1}^{\infty} \beta_{n}=\infty$;

(ii) $0<\tau<\frac{\bar{\tau}}{\mu}$;

(iii) $0<a<\xi_{n}<\eta_{n}<b<\frac{1}{1+\sqrt{1+L^{2}}}, \forall n \geq 1$.

Then, the sequence $\left\{x_{n}\right\}$ generated by 3.1 is bounded.

Proof. Let $p \in \Gamma$. We have $p=T_{r_{n}}^{F_{1}}\left(I-r_{n} f_{1}\right) p$ and $A p=T_{s_{n}}^{F_{2}}\left(I-s_{n} f_{2}\right) A p$. From Lemma 2.2, we have that $\left(T_{r_{n}}^{F_{1}}\left(I-r f_{1}\right)\right)$ and $\left(T_{s_{n}}^{F_{2}}\left(I-s_{n} f_{2}\right)\right)$ are nonexpansive mappings. Using (3.1) and (3.2), we obtain

$$
\begin{aligned}
\left\|u_{n}-p\right\|^{2}= & \| T_{r_{n}}^{F_{1}}\left(I-r_{n} f_{1}\right)\left(x_{n}+\gamma_{n} A^{*}\left(T_{s_{n}}^{F_{2}}\left(I-s_{n} f_{2}\right) A x_{n}\right)-T_{r_{n}}^{F_{1}}\left(I-r_{n} f_{1}\right) p \|^{2}\right. \\
\leq & \left.\| x_{n}+\gamma_{n} A^{*}\left(T_{s_{n}}^{F_{2}}\left(I-s_{n} f_{2}\right)-I\right) A x_{n}\right)-p \|^{2} \\
\leq & \left\|x_{n}-p\right\|^{2}+\gamma_{n}^{2}\left\|A^{*}\left(T_{s_{n}}^{F_{2}}\left(I-s_{n} f_{2}\right)-I\right) A x_{n}\right\|^{2} \\
& +2 \gamma_{n}\left\langle x_{n}-p, A^{*}\left(T_{s_{n}}^{F_{2}}\left(I-s_{n} f_{2}\right)-I\right) A x_{n}\right\rangle
\end{aligned}
$$


where

$$
\begin{aligned}
2 \gamma_{n}\left\langle x_{n}-\right. & \left.p, A^{*}\left(T_{s_{n}}^{F_{2}}\left(I-s_{n} f_{2}\right)-I\right) A x_{n}\right\rangle \\
= & 2 \gamma_{n}\left\langle A\left(x_{n}-p\right),\left(T_{s_{n}}^{F_{2}}\left(I-s_{n} f_{2}\right)-I\right) A x_{n}\right\rangle \\
= & 2 \gamma_{n}\left\langle A\left(x_{n}-p\right)+\left(T_{s_{n}}^{F_{2}}\left(I-s_{n} f_{2}\right)-I\right) A x_{n}\right. \\
& \left.-\left(T_{s_{n}}^{F_{2}}\left(I-s_{n} f_{2}\right)-I\right) A x_{n},\left(T_{s_{n}}^{F_{2}}\left(I-s_{n} f_{2}\right)-I\right) A x_{n}\right\rangle \\
= & 2 \gamma_{n}\left\{\left\langle T_{s_{n}}^{F_{2}}\left(I-s_{n} f_{2}\right) A x_{n}-A p,\left(T_{s_{n}}^{F_{2}}\left(I-s f_{2}\right)-I\right) A x_{n}\right\rangle\right. \\
& \left.-\left\|\left(T_{s_{n}}^{F_{2}}\left(I-s_{n} f_{2}\right)-I\right) A x_{n}\right\|^{2}\right\} \\
\leq & \left.2 \gamma_{n}\left\{\frac{1}{2} \| T_{s_{n}}^{F_{2}}\left(I-s_{n} f_{2}\right)-I\right) A x_{n}\left\|^{2}-\right\|\left(T_{s_{n}}^{F_{2}}\left(I-s_{n} f_{2}\right)-I\right) A x_{n} \|^{2}\right\} \\
\leq & -\gamma_{n}\left\|\left(T_{s_{n}}^{F_{2}}\left(I-s_{n} f_{2}\right)-I\right) A x_{n}\right\|^{2} .
\end{aligned}
$$

On substituting (3.4) into (3.3), we have

$$
\begin{aligned}
\left\|u_{n}-p\right\|^{2} \leq & \left\|x_{n}-p\right\|^{2}+\gamma_{n}^{2}\left\|A^{*}\left(T_{s_{n}}^{F_{2}}\left(I-s_{n} f_{2}\right)-I\right) A x_{n}\right\|^{2} \\
& -\gamma_{n}\left\|\left(T_{s_{n}}^{F_{2}}\left(I-s_{n} f_{2}\right)-I\right) A x_{n}\right\|^{2} \\
= & \left\|x_{n}-p\right\|^{2}-\gamma_{n}\left[\left\|\left(T_{s_{n}}^{F_{2}}\left(I-s_{n} f_{2}\right)-I\right) A x_{n}\right\|^{2}\right. \\
& \left.-\gamma_{n}\left\|\left(T_{s_{n}}^{F_{2}}\left(I-s_{n} f_{2}\right)-I\right) A x_{n}\right\|^{2}\right] .
\end{aligned}
$$

Since $\gamma_{n} \in\left(\epsilon, \frac{\left\|\left(T_{s_{n}}^{F_{2}}\left(I-s_{n} f_{2}\right)-I\right) A x_{n}\right\|^{2}}{\left\|A^{*}\left(T_{s_{n}}^{F_{2}}\left(I-s_{n} f_{2}\right)-I\right) A x_{n}\right\|^{2}}-\epsilon\right)$, we obtain

$$
\left\|u_{n}-p\right\|^{2} \leq\left\|x_{n}-p\right\|^{2} .
$$

Using (3.1) and (3.6), we have

$$
\begin{aligned}
\left\|y_{n}-p\right\|^{2} & =\left\|\alpha_{n} u_{n}+\left(1-\alpha_{n}\right)\left(1-\xi_{n}\right) I+\xi_{n} T\left(1-\eta_{n}\right) I+\eta_{n} T\left(u_{n}-p\right)\right\|^{2} \\
& =\left\|\alpha_{n}\left(u_{n}-p\right)+\left(1-\alpha_{n}\right)\left(K u_{n}-p\right)\right\|^{2} \\
& \leq \alpha_{n}\left\|u_{n}-p\right\|^{2}+\left(1-\alpha_{n}\right)\left\|K u_{n}-p\right\|^{2}-\alpha\left(1-\alpha_{n}\right)\left\|K u_{n}-u_{n}\right\|^{2} \\
& \leq \alpha_{n}\left\|u_{n}-p\right\|^{2}+\left(1-\alpha_{n}\right)\left\|u_{n}-p\right\|^{2} \\
& \leq\left\|u_{n}-p\right\|^{2} \\
& \leq\left\|x_{n}-p\right\|^{2} .
\end{aligned}
$$

Furthermore, using Lemma 2.4, (3.1), and (3.7), we obtain

$$
\begin{aligned}
\left\|x_{n+1}-p\right\| & =\left\|\beta_{n}\left[\tau g\left(x_{n}\right)-D p\right]+\left(1-\beta_{n} D\right)\left(y_{n}-p\right)\right\| \\
& \leq\left(1-\beta_{n} \bar{\tau}\right)\left\|y_{n}-p\right\|+\beta_{n}\left\|\tau g\left(x_{n}\right)-D p\right\| \\
& \leq\left(1-\beta_{n} \bar{\tau}\right)\left\|y_{n}-p\right\|+\beta_{n}\left[\left\|\tau g\left(x_{n}\right)-\tau g(p)\right\|+\|\tau g(p)-D p\|\right] \\
& \leq\left[1-(\bar{\tau}-\tau \mu) \beta_{n}\right]\left\|x_{n}-p\right\|+\beta_{n}\|\tau g(p)-D p\| .
\end{aligned}
$$


It follows from induction that

$$
\left\|x_{n}-p\right\| \leq \max \left\{\left\|x_{1}-p\right\|, \frac{\|\tau g(p)-D p\|}{\bar{\tau}-\tau \mu}\right\}, \quad n \geq 1
$$

Hence $\left\{x_{n}\right\}$ is bounded. Consequently, we deduce that $\left\{u_{n}\right\}$ and $\left\{y_{n}\right\}$ are all bounded.

Theorem 3.3. Let $C$ and $Q$ be nonempty closed convex subsets of real Hilbert spaces $H_{1}$ and $H_{2}$ respectively. Let $A: H_{1} \rightarrow H_{2}$ be a bounded linear operator and $\mathrm{T}: \mathrm{H}_{1} \rightarrow \mathrm{H}_{2}$ be an L-Lipschitzian and quasi-pseudocontractive mapping with $L \geq 1$. Let $F_{1}: C \times C \rightarrow \mathbb{R}, F_{2}: Q \times Q \rightarrow \mathbb{R}$ be bifunctions satisfying (A1)-(A4) and let $D$ be a strongly positive bounded linear operator on $H_{1}$ with coefficient $\bar{\tau}>0$. Let $f_{1}: H_{1} \rightarrow H_{1}$ be a $\rho_{1}$-inverse strongly monotone mapping and $f_{2}: H_{2} \rightarrow H_{2}$ be a $\rho_{2}$-inverse strongly monotone mapping. Assume that $\Gamma:=F(T) \cap \Omega \neq \emptyset$ and that $g$ is a contraction mapping with coefficient $\mu \in(0,1)$. Let $K:=\left(\left(1-\xi_{n}\right) I+\right.$ $\xi_{n} T\left(\left(1-\eta_{n}\right) I+\eta_{n} T\right), 0<r \leq r_{n}<2 \rho_{1}, 0<s \leq s_{n}<2 \rho_{2}, \alpha_{n} \in(0,1)$, and the step size $\gamma_{n}$ is chosen in such a way that, for some $\epsilon>0$,

$$
\gamma_{n} \in\left(\epsilon, \frac{\left\|\left(T_{s_{n}}^{F_{2}}\left(I-s_{n} f_{2}\right)-I\right) A x_{n}\right\|^{2}}{\left\|A^{*}\left(T_{s_{n}}^{F_{2}}\left(I-s_{n} f_{2}\right)-I\right) A x_{n}\right\|^{2}}-\epsilon\right),
$$

for all $T_{s_{n}}^{F_{2}}\left(I-s_{n} f_{2}\right) A x_{n} \neq A x_{n}$ and $\gamma_{n}=\gamma$ otherwise $(\gamma$ being any nonnegative real number), with the sequences $\left\{\beta_{n}\right\},\left\{\xi_{n}\right\},\left\{\eta_{n}\right\}$ satisfying the following conditions:

(i) $\beta_{n} \in(0,1), \lim _{n \rightarrow \infty} \beta_{n}=0$, and $\sum_{n=1}^{\infty} \beta_{n}=\infty$;

(ii) $0<a<\xi_{n}<\eta_{n}<b<\frac{1}{1+\sqrt{1+L^{2}}}, \forall n \geq 1$;

(iii) $0<\tau<\frac{\bar{\tau}}{\mu}$;

(iv) $0<\liminf _{n \rightarrow \infty} \alpha_{n} \leq \limsup _{n \rightarrow \infty} \alpha_{n}<1$.

Then, the sequence $\left\{x_{n}\right\}$ generated by 3.1 converges strongly to $x^{*} \in \Gamma$ which solves the variational inequality

$$
\left\langle(D-\tau g) x^{*}, x^{*}-x\right\rangle \leq 0, \forall x \in \Gamma
$$

where $x=P_{\Gamma}(I+\tau g-D) x$. 
Proof. Let $p \in \Gamma$. Then, applying Lemma 2.4 and 3.5 , we obtain

$$
\begin{aligned}
\left\|x_{n+1}-p\right\|^{2}= & \left\|\beta_{n}\left[\tau g\left(x_{n}\right)-D p\right]+\left(I-\beta_{n} D\right)\left(y_{n}-p\right)\right\|^{2} \\
\leq & \left(1-\beta_{n} \bar{\tau}\right)^{2}\left\|y_{n}-p\right\|^{2}+\beta_{n}^{2}\left\|\tau g\left(x_{n}\right)-D p\right\|^{2} \\
& +2 \beta_{n}\left(1-\beta_{n} \bar{\tau}\right)\left\|\tau g\left(x_{n}\right)-D p\right\|\left\|y_{n}-p\right\| \\
\leq & \left(1-\beta_{n} \bar{\tau}\right)^{2}\left\|u_{n}-p\right\|^{2}+\beta_{n}^{2}\left\|\tau g\left(x_{n}\right)-D p\right\|^{2} \\
& +2 \beta_{n}\left(1-\beta_{n} \bar{\tau}\right)\left\|\tau g\left(x_{n}\right)-D p\right\|\left\|y_{n}-p\right\| \\
\leq & \left(1-\beta_{n} \bar{\tau}\right)^{2}\left[\left\|x_{n}-p\right\|^{2}+\gamma_{n}^{2}\left\|A^{*}\left(T_{s_{n}}^{F_{2}}\left(I-s_{n} f_{2}\right)-I\right) A x_{n}\right\|^{2}\right. \\
& \left.-\gamma_{n}\left\|\left(T_{s_{n}}^{F_{2}}\left(I-s_{n} f_{2}\right)-I\right) A x_{n}\right\|^{2}\right]+\beta_{n}^{2}\left\|\tau g\left(x_{n}\right)-D p\right\| \\
& +2 \beta_{n}\left(1-\beta_{n} \bar{\tau}\right)\left\|\tau g\left(x_{n}\right)-D p\right\|\left\|y_{n}-p\right\| \\
\leq & \left(1-\beta_{n} \bar{\tau}\right)^{2}\left\|x_{n}-p\right\|^{2}-\gamma_{n}\left[\left\|\left(T_{s_{n}}^{F_{2}}\left(I-s_{n} f_{2}\right)-I\right) A x_{n}\right\|^{2}\right. \\
& \left.-\gamma_{n}\left\|A^{*}\left(T_{s_{n}}^{F_{2}}\left(I-s_{n} f_{2}\right)-I\right) A x_{n}\right\|^{2}\right] \\
& +\beta_{n}^{2}\left\|\tau g\left(x_{n}\right)-D p\right\|^{2}+2 \beta_{n}\left(1-\beta_{n} \bar{\tau}\right)\left\|\tau g\left(x_{n}\right)-D p\right\|\left\|y_{n}-p\right\|
\end{aligned}
$$

It follows from (3.8) and the condition $\gamma_{n} \in\left(\epsilon, \frac{\left\|\left(T_{s_{n}}^{F_{2}}\left(I-s_{n} f_{2}\right)-I\right) A x_{n}\right\|^{2}}{\left\|A^{*}\left(T_{s_{n}}^{F_{2}}\left(I-s_{n} f_{2}\right)-I\right) A x_{n}\right\|^{2}}-\epsilon\right)$ that

$$
\begin{aligned}
\left\|x_{n+1}-p\right\|^{2} \leq & \left(1-\beta_{n} \bar{\tau}\right)^{2}\left\|x_{n}-p\right\|^{2}-\epsilon\left\|A^{*}\left(T_{s_{n}}^{F_{2}}\left(I-s_{n} f_{2}\right)-I\right) A x_{n}\right\|^{2} \\
& +\beta_{n}^{2}\left\|\tau g\left(x_{n}\right)-D p\right\|^{2}+2 \beta_{n}\left(1-\beta_{n} \bar{\tau}\right)\left\|\tau g\left(x_{n}\right)-D p\right\|\left\|y_{n}-p\right\| .
\end{aligned}
$$

We now divide our proof into two cases.

Case 1: Assume that $\left\{\left\|x_{n}-p\right\|\right\}$ is a monotonically nonincreasing sequence. Then $\left\{x_{n}\right\}$ is convergent and clearly

$$
\lim _{n \rightarrow \infty}\left\|x_{n}-p\right\|=\lim _{n \rightarrow \infty}\left\|x_{n+1}-p\right\| .
$$

Thus from 3.9$)$ we have that

$$
\begin{aligned}
\epsilon \| A^{*}\left(T_{s_{n}}^{F_{2}}(I-\right. & \left.\left.s_{n} f_{2}\right)-I\right) A x_{n}\left\|^{2} \leq\left(1-\beta_{n} \bar{\tau}\right)^{2}\right\| x_{n}-p\left\|^{2}-\right\| x_{n+1}-p \|^{2} \\
& +\beta_{n}^{2}\left\|\tau g\left(x_{n}\right)-D p\right\|^{2}+2 \beta_{n}\left(1-\beta_{n} \bar{\tau}\right)\left\|\tau g\left(x_{n}\right)-D p\right\|\left\|y_{n}-p\right\| .
\end{aligned}
$$

Hence, from condition (i) of Theorem 3.3 we obtain

$$
\lim _{n \rightarrow \infty} \| A^{*}\left(T_{s_{n}}^{F_{2}}\left(I-s_{n} f_{2}\right) A x_{n} \|=0 .\right.
$$

Furthermore, from 3.8 and 3.10 we have that

$$
\begin{aligned}
\gamma_{n} \|\left(T_{s_{n}}^{F_{2}}\left(I-s_{n} f_{2}\right)-\right. & I) A x_{n}\left\|^{2} \leq\left(1-\beta_{n} \bar{\tau}\right)^{2}\right\| x_{n}-p\left\|^{2}-\right\| x_{n+1}-p \|^{2} \\
& +\gamma_{n}^{2}\left\|A^{*}\left(T_{s_{n}}^{F-2}\left(I-s_{n} f_{2}\right)-I\right) A x_{n}\right\|^{2}+\beta_{n}^{2}\left\|\tau g\left(x_{n}\right)-D p\right\|^{2} \\
& +2 \beta_{n}\left(1-\beta_{n} \bar{\tau}\right)\left\|\tau g\left(x_{n}\right)-D p\right\|\left\|y_{n}-p\right\|,
\end{aligned}
$$


which implies from condition (i) of Theorem 3.3 and 3.2 that

$$
\lim _{n \rightarrow \infty}\left\|\left(T_{s_{n}}^{F_{2}}\left(I-s_{n} f_{2}\right)-I\right) A x_{n}\right\|=0 .
$$

Let $w_{n}=x_{n}+\gamma_{n} A^{*}\left(T_{s_{n}}^{F_{2}}\left(I-s_{n} f_{2}\right)-I\right) A x_{n}$. Applying inequality 3.6, we have

$$
\left\|w_{n}-p\right\| \leq\left\|x_{n}-p\right\|
$$

Using the property of inverse strongly monotone operator and 3.12 , we have

$$
\begin{aligned}
\left\|u_{n}-p\right\|^{2} & =\left\|T_{r_{n}}^{F_{1}}\left(I-r_{n} f_{1}\right) w_{n}-T_{r_{n}}^{F_{1}}\left(I-r_{n} f_{1}\right) p\right\|^{2} \\
& \leq\left\|\left(I-r_{n} f_{1}\right) w_{n}-\left(I-r_{n} f_{1}\right) p\right\|^{2} \\
& =\left\|w_{n}-p\right\|^{2}-2 r_{n}\left\langle w_{n}-p, f_{1} w_{n}-f_{1} p\right\rangle+r_{n}^{2}\left\|f_{1} w_{n}-f_{1} p\right\|^{2} \\
& \leq\left\|x_{n}-p\right\|^{2}-r_{n}\left(2 \rho_{1}-r_{n}\right)\left\|f_{1} w_{n}-f_{1} p\right\|^{2} .
\end{aligned}
$$

From Theorem 3.3 , we have that

$$
\begin{aligned}
\left\|x_{n+1}-p\right\|^{2}= & \left\|\beta_{n}\left[\tau g\left(x_{n}\right)-D p\right]+\left(I-\beta_{n} D\right)\left(y_{n}-p\right)\right\|^{2} \\
\leq & \left(1-\beta_{n} \bar{\tau}\right)^{2}\left\|y_{n}-p\right\|^{2}+\beta_{n}^{2}\left\|\tau g\left(x_{n}\right)-D p\right\|^{2} \\
& +2 \beta_{n}\left(1-\beta_{n} \bar{\tau}\right)\left\|\tau g\left(x_{n}\right)-D p\right\|\left\|y_{n}-p\right\| \\
\leq & \left(1-\beta_{n} \bar{\tau}\right)^{2}\left\|u_{n}-p\right\|^{2}+\beta_{n}^{2}\left\|\tau g\left(x_{n}\right)-D p\right\|^{2} \\
& +2 \beta_{n}\left(1-\beta_{n} \bar{\tau}\right)\left\|\tau g\left(x_{n}\right)-D p\right\|\left\|y_{n}-p\right\| .
\end{aligned}
$$

Substituting (3.13) into 3.14, we obtain

$$
\begin{aligned}
\left\|x_{n+1}-p\right\|^{2} \leq & \left(1-\beta_{n} \bar{\tau}\right)^{2}\left[\left\|x_{n}-p\right\|^{2}-r_{n}\left(2 \rho_{1}-r_{n}\right)\left\|f_{1} w_{n}-f_{1} p\right\|^{2}\right] \\
& +\beta_{n}^{2}\left\|\tau g\left(x_{n}\right)-D p\right\|^{2} \\
& +2 \beta_{n}\left(1-\beta_{n} \bar{\tau}\right)\left\|\tau g\left(x_{n}\right)-D p\right\|\left\|y_{n}-p\right\| \\
\leq & \left(1-\beta_{n} \bar{\tau}\right)^{2}\left\|x_{n}-p\right\|^{2}-r_{n}\left(2 \rho_{1}-r_{n}\right)\left\|f_{1} w_{n}-f_{1} p\right\|^{2} \\
& +\beta_{n}^{2}\left\|\tau g\left(x_{n}\right)-D p\right\|^{2} \\
& +2 \beta_{n}\left(1-\beta_{n} \bar{\tau}\right)\left\|\tau g\left(x_{n}\right)-D p\right\|\left\|y_{n}-p\right\| .
\end{aligned}
$$

Hence,

$$
\begin{aligned}
r_{n}\left(2 \rho_{1}-r_{n}\right)\left\|f_{1} w_{n}-f_{1} p\right\|^{2} \leq & \left(1-\beta_{n} \bar{\tau}\right)^{2}\left\|x_{n}-p\right\|^{2}-\left\|x_{n+1}-p\right\|^{2} \\
& +\beta_{n}^{2}\left\|\tau g\left(x_{n}\right)-D p\right\|^{2} \\
& +2 \beta_{n}\left(1-\beta_{n} \bar{\tau}\right)\left\|\tau g\left(x_{n}\right)-D p\right\|\left\|y_{n}-p\right\|
\end{aligned}
$$

Therefore, from condition (i) of Lemma 3.2 we obtain

$$
\lim _{n \rightarrow \infty}\left\|f_{1} w_{n}-f_{1} p\right\|=0 .
$$


By the firm nonexpansivity of $T_{r_{n}}^{F_{1}}$, we have

$$
\begin{aligned}
\left\|u_{n}-p\right\|^{2}= & \left\|T_{r_{n}}^{F_{1}}\left(I-r_{n} f_{1}\right) w_{n}-T_{r_{n}}^{F_{1}}\left(I-r_{n} f_{1}\right) p\right\|^{2} \\
\leq & \left\langle u_{n}-p,\left(I-r_{n} f_{1}\right) w_{n}-\left(I-r_{n} f_{1}\right) p\right\rangle \\
= & \frac{1}{2}\left(\left\|u_{n}-p\right\|^{2}+\left\|\left(I-r_{n} f_{1}\right) w_{n}-\left(I-r_{n} f_{1}\right) p\right\|^{2}\right. \\
& \left.-\left\|\left(u_{n}-p\right)-\left(I-r_{n} f_{1}\right) w_{n}+\left(I-r_{n} f_{1}\right) p\right\|^{2}\right) .
\end{aligned}
$$

That is,

$$
\begin{aligned}
\left\|u_{n}-p\right\|^{2} \leq & \left\|\left(I-r_{n} f_{1}\right) w_{n}-\left(I-r_{n} f_{1}\right) p\right\|^{2}-\left\|\left(u_{n}-w_{n}\right)+r_{n}\left(f_{1} w_{n}-f_{1} p\right)\right\|^{2} \\
\leq & \left\|w_{n}-p\right\|^{2} \\
& -\left(\left\|u_{n}-w_{n}\right\|^{2}+2 r_{n}\left\langle u_{n}-w_{n}, f_{1} w_{n}-f_{1} p\right\rangle+r_{n}^{2}\left\|f_{1} w_{n}-f_{1} p\right\|^{2}\right) \\
\leq & \left\|w_{n}-p\right\|^{2} \\
& -\left\|u_{n}-w_{n}\right\|^{2}+2 r_{n}\left\|u_{n}-w_{n}\right\|\left\|F_{1} w_{n}-f_{1} p\right\|-r_{n}^{2}\left\|f_{1} w_{n}-f_{1} p\right\|^{2} .
\end{aligned}
$$

From 3.8, 3.12 and 3.16, we obtain

$$
\begin{aligned}
\left\|x_{n+1}-p\right\|^{2} \leq & \left(1-\beta_{n} \bar{\tau}\right)^{2}\left\|u_{n}-p\right\|^{2}+\beta_{n}^{2}\left\|\tau g\left(x_{n}\right)-D p\right\|^{2} \\
& +2 \beta_{n}\left(1-\beta_{n} \bar{\tau}\right)\left\|\tau g\left(x_{n}\right)-D p\right\|\left\|y_{n}-p\right\| \\
\leq & \left(1-\beta_{n} \bar{\tau}\right)^{2}\left[\left\|w_{n}-p\right\|^{2}-\left\|u_{n}-w_{n}\right\|^{2}+2 r_{n}\left\|u_{n}-w_{n}\right\|\left\|f_{1} w_{n}-f_{1} p\right\|\right. \\
& \left.-r_{n}^{2}\left\|f_{1} w_{n}-f_{1} p\right\|^{2}\right]+\beta_{n}^{2}\left\|\tau g\left(x_{n}\right)-D p\right\|^{2} \\
& +2 \beta_{n}\left(1-\beta_{n} \bar{\tau}\right)\left\|\tau g\left(x_{n}\right)-D p\right\|\left\|y_{n}-p\right\| \\
\leq & \left(1-2 \beta_{n} \bar{\tau}+\left(\beta_{n} \bar{\tau}\right)\right)\left\|x_{n}-p\right\|^{2}-\left(1-\beta_{n} \bar{\tau}\right)^{2}\left\|u_{n}-w_{n}\right\|^{2} \\
& +2 r_{n}\left(1-\beta_{n} \bar{\tau}\right)^{2}\left\|u_{n}-w_{n}\right\|\left\|f_{1} w_{n}-f_{1} p\right\| \\
& -\left(1-\beta_{n} \bar{\tau}\right)^{2} r_{n}^{2}\left\|f_{1} w_{n}-f_{1} p\right\|^{2} \\
& +\beta_{n}^{2}\left\|\tau g\left(x_{n}\right)-D p\right\|^{2}+2 \beta_{n}\left(1-\beta_{n} \bar{\tau}\right)\left\|\tau g\left(x_{n}\right)-D p\right\|\left\|y_{n}-p\right\| \\
\leq & \left\|x_{n}-p\right\|^{2}+\left(\beta_{n} \bar{\tau}\right)^{2}\left\|x_{n}-p\right\|^{2}-\left(1-\beta_{n} \bar{\tau}\right)^{2}\left\|u_{n}-w_{n}\right\|^{2} \\
& +2 r_{n}\left(1-\beta_{n} \bar{\tau}\right)^{2}\left\|u_{n}-w_{n}\right\|\left\|f_{1} w_{n}-f_{1} p\right\| \\
& -\left(1-\beta_{n} \bar{\tau}\right)^{2} r_{n}^{2}\left\|f_{1} w_{n}-f_{1} p\right\|^{2}+\beta_{n}^{2}\left\|\tau g\left(x_{n}\right)-D p\right\|^{2} \\
& +2 \beta_{n}\left(1-\beta_{n} \bar{\tau}\right)\left\|\tau g\left(x_{n}\right)-D p\right\|\left\|y_{n}-p\right\|,
\end{aligned}
$$

which yields

$$
\begin{aligned}
\left(1-\beta_{n} \bar{\tau}\right)^{2} & \left\|u_{n}-w_{n}\right\|^{2} \leq\left\|x_{n}-p\right\|^{2}-\left\|x_{n+1}-p\right\|^{2}+\left(\beta_{n} \bar{\tau}\right)^{2}\left\|x_{n}-p\right\|^{2} \\
& +2 r_{n}\left(1-\beta_{n} \bar{\tau}\right)^{2}\left\|u_{n}-w_{n}\right\|\left\|f_{1} w_{n}-f_{1} p\right\|-\left(1-\beta_{n} \bar{\tau}\right)^{2} r_{n}^{2}\left\|f_{1} w_{n}-f_{1} p\right\|^{2} \\
& +\beta_{n}^{2}\left\|\tau g\left(x_{n}\right)-D p\right\|^{2}+2 \beta_{n}(1-\beta \bar{\tau})\left\|\tau g\left(x_{n}\right)-D p\right\|\left\|y_{n}-p\right\| .
\end{aligned}
$$


From condition (i) of Lemma 3.2 and (3.15), we obtain

$$
\lim _{n \rightarrow \infty}\left\|u_{n}-w_{n}\right\|=0 .
$$

Since $w_{n}=x_{n}+\gamma_{n} A^{*}\left(T_{s_{n}}^{F_{2}}\left(I-s_{n} f_{2}\right)-I\right) A x_{n}$, we have that

$$
\begin{aligned}
\left\|w_{n}-x_{n}\right\| & =\left\|x_{n}+\gamma_{n} A^{*}\left(T_{s_{n}}^{F_{2}}\left(I-s_{n} f_{2}\right)-I\right) A x_{n}-x_{n}\right\| \\
& \leq \gamma_{n}\left\|A^{*}\left(T_{s_{n}}^{F_{2}}\left(I-s_{n} f_{2}\right)-I\right) A x_{n}\right\|,
\end{aligned}
$$

which implies from 3.10 that

$$
\lim _{n \rightarrow \infty}\left\|w_{n}-x_{n}\right\|=0 .
$$

From 3.1), 3.17), and 3.18, we obtain

$$
\left\|u_{n}-x_{n}\right\| \leq\left\|u_{n}-w_{n}\right\|+\left\|w_{n}-x_{n}\right\| \rightarrow 0, \quad n \rightarrow \infty .
$$

Again,

$$
\begin{aligned}
\left\|y_{n}-p\right\|^{2} & =\left\|\alpha_{n}\left(u_{n}-p\right)+\left(1-\alpha_{n}\right)\left(K u_{n}-p\right)\right\|^{2} \\
& \leq \alpha_{n}\left\|u_{n}-p\right\|^{2}+\left(1-\alpha_{n}\right)\left\|K u_{n}-p\right\|^{2}-\alpha\left(1-\alpha_{n}\right)\left\|K u_{n}-u_{n}\right\|^{2} \\
& \leq \alpha_{n}\left\|u_{n}-p\right\|^{2}+\left(1-\alpha_{n}\right)\left\|u_{n}-p\right\|^{2}-\alpha\left(1-\alpha_{n}\right)\left\|K u_{n}-u_{n}\right\|^{2} \\
& =\left\|u_{n}-p\right\|^{2}-\alpha_{n}\left(1-\alpha_{n}\right)\left\|K u_{n}-u_{n}\right\|^{2} .
\end{aligned}
$$

From 3.1), we have

$$
\begin{aligned}
\left\|x_{n+1}-p\right\|^{2} \leq & \left(1-\beta_{n} \bar{\tau}\right)^{2}\left\|y_{n}-p\right\|^{2}+\beta_{n}\left\|\tau g\left(x_{n}\right)-D p\right\|^{2} \\
& +2 \beta_{n}\left(1-\beta_{n} \bar{\tau}\right)\left\|\tau g\left(x_{n}\right)-D p\right\|\left\|y_{n}-p\right\| .
\end{aligned}
$$

On substituting 3.20 into 3.21 , we obtain

$$
\begin{aligned}
\left\|x_{n+1}-p\right\|^{2} \leq & \left(1-\beta_{n} \bar{\tau}\right)^{2}\left[\left\|u_{n}-p\right\|^{2}-\alpha_{n}\left(1-\alpha_{n}\right)\left\|K u_{n}-u_{n}\right\|^{2}\right] \\
& +\beta_{n}\left\|\tau g\left(x_{n}\right)-D p\right\|^{2}+2 \beta_{n}\left(1-\beta_{n} \bar{\tau}\right)\left\|\tau g\left(x_{n}\right)-D p\right\|\left\|y_{n}-p\right\| \\
\leq & \left(1-\beta_{n} \bar{\tau}\right)^{2}\left\|x_{n}-p\right\|^{2}-\alpha_{n}\left(1-\alpha_{n}\right)\left(1-\beta_{n} \bar{\tau}\right)\left\|K u_{n}-u_{n}\right\|^{2} \\
& +\beta_{n}\left\|\tau g\left(x_{n}\right)-D p\right\|^{2}+2 \beta_{n}\left(1-\beta_{n} \bar{\tau}\right)\left\|\tau g\left(x_{n}\right)-D p\right\|\left\|y_{n}-p\right\|,
\end{aligned}
$$

which yields

$$
\begin{gathered}
\alpha_{n}\left(1-\alpha_{n}\right)\left(1-\beta_{n} \bar{\tau}\right)^{2}\left\|K u_{n}-u_{n}\right\|^{2} \leq\left(1-\beta_{n} \bar{\tau}\right)^{2}\left\|x_{n}-p\right\|^{2}-\left\|x_{n+1}-p\right\|^{2} \\
+\beta_{n}\left\|\tau g\left(x_{n}\right)-D p\right\|^{2}+2 \beta_{n}\left(1-\beta_{n} \bar{\tau}\right)\left\|\tau g\left(x_{n}\right)-D p\right\|\left\|y_{n}-p\right\| .
\end{gathered}
$$

Thus, from condition (i) of Lemma 3.2 , we obtain

$$
\lim _{n \rightarrow \infty}\left\|K u_{n}-u_{n}\right\|=0 .
$$

Also, from 3.1 we have

$$
\begin{aligned}
\left\|y_{n}-u_{n}\right\| & =\left\|\alpha_{n} u_{n}+\left(1-\alpha_{n}\right) K u_{n}-u_{n}\right\| \\
& \leq\left(1-\alpha_{n}\right)\left\|K u_{n}-u_{n}\right\|,
\end{aligned}
$$


which implies that

$$
\lim _{n \rightarrow \infty}\left\|y_{n}-u_{n}\right\|=0
$$

Again, from (3.19) and 3.24) we obtain

$$
\left\|y_{n}-x_{n}\right\| \leq\left\|y_{n}-u_{n}\right\|+\left\|u_{n}-x_{n}\right\| \rightarrow 0, \quad n \rightarrow \infty .
$$

From 3.1 we have

$$
\begin{aligned}
\left\|x_{n+1}-x_{n}\right\| & \leq\left\|x_{n+1}-y_{n}\right\|+\left\|y_{n}-x_{n}\right\| \\
& =\left\|\beta_{n} \tau g\left(x_{n}\right)+\left(I-\beta_{n} D\right) y_{n}-y_{n}\right\|+\left\|y_{n}-x_{n}\right\| \\
& \leq \beta_{n}\left\|\tau g\left(x_{n}\right)-D y_{n}\right\|+\left\|y_{n}-x_{n}\right\| .
\end{aligned}
$$

From condition (i) of Lemma 3.2 and 3.25 we have that

$$
\lim _{n \rightarrow \infty}\left\|x_{n+1}-x_{n}\right\|=0 .
$$

Since $\left\{x_{n}\right\}$ is bounded, there exists a weakly convergent subsequence $\left\{x_{n_{j}}\right\}$ of $\left\{x_{n}\right\}$ such that $x_{n_{j}} \rightarrow x^{*}$. Since every Hilbert space has the Opial property, we have $x_{n} \rightarrow x^{*}$. On the other hand, from $(3.19)$ we have that $u_{n} \rightarrow x^{*}$. Using (3.23 and the demiclosedness property of $K$, we have that $K x^{*}=x^{*}$. Hence $x^{*} \in F(T)$. Next, we show that $x^{*} \in \Omega$. Assume that $x^{*} \notin E P\left(F_{1}, f_{1}\right)$; since $E P\left(F_{1}, f_{1}\right)=F\left(T_{r}^{F_{1}}\left(I-r f_{1}\right)\right)$, we obtain $x^{*} \neq T_{r}^{F_{1}}\left(I-r f_{1}\right) x^{*}$. Using Opial's condition and (3.17), Lemma 2.3, and Lemma 3.1 we obtain

$$
\begin{aligned}
\liminf _{j \rightarrow \infty}\left\|w_{n_{j}}-x^{*}\right\| & <\liminf _{j \rightarrow \infty}\left\|w_{n_{j}}-T_{r}^{F_{1}}\left(I-r f_{1}\right) x^{*}\right\| \\
\leq & \liminf _{j \rightarrow \infty}\left(\left\|w_{n_{j}}-T_{r_{n}}^{F_{1}}\left(I-r_{n} f_{1}\right) w_{n_{j}}\right\|\right. \\
& \quad+\left\|T_{r_{n}}^{F_{1}}\left(I-r_{n} f_{1}\right) w_{n_{j}}-T_{r}^{F_{1}}\left(I-r f_{1}\right) w_{n_{j}}\right\| \\
& \left.\quad+\left\|T_{r}^{F_{1}}\left(I-r f_{1}\right) w_{n_{j}}-T_{r}^{F_{1}}\left(I-r f_{1}\right) x^{*}\right\|\right) \\
\leq & \liminf _{j \rightarrow \infty}\left(\left\|w_{n_{j}}-u_{n_{j}}\right\|+\left\|w_{n_{j}}-x^{*}\right\|\right) \\
\leq & \liminf _{j \rightarrow \infty}\left\|w_{n_{j}}-x^{*}\right\| .
\end{aligned}
$$

This is a contradiction, therefore $x^{*} \in E P\left(F_{1}, f_{1}\right)$.

Next, we show that $A x^{*} \in E P\left(F_{2}, f_{2}\right)$. Since $A$ is a bounded linear operator, $A x_{n_{j}} \rightarrow A x^{*}$ as $j \rightarrow \infty$. Assume that $A x^{*} \notin E P\left(F_{2}, f_{2}\right)$ and since $\operatorname{EP}\left(F_{2}, f_{2}\right)=$ $F\left(T_{s_{n}}^{F_{2}}\left(I-s_{n} f_{2}\right)\right)$, we obtain $A x^{*} \neq T_{s_{n}}^{F_{2}}\left(I-s_{n} f_{2}\right) A x^{*}$. Using Opial's condition and (3.11), we obtain, by a similar argument to that given above, $A x^{*} \in E P\left(F_{2}, f_{2}\right)$. Hence, we conclude that $x^{*} \in \Omega$. Therefore, $x^{*} \in \Gamma$.

We now show that $\limsup _{j \rightarrow \infty}\left\langle(D-\tau g) x, x-x_{n}\right\rangle \leq 0$, where $x=P_{\Gamma}(I+\tau g-$ $D) x$. Indeed, the subsequence $\left\{x_{n_{j}}\right\}$ of $\left\{x_{n}\right\}$ converges weakly to $x^{*}$. We obtain, 
by the property of metric projection $P_{\Gamma}$,

$$
\begin{aligned}
\limsup _{n \rightarrow \infty}\left\langle(D-\tau g) x, x-x_{n}\right\rangle & =\lim _{j \rightarrow \infty}\left\langle(D-\tau g) x, x-x_{n_{j}}\right\rangle \\
& =\left\langle(D-\tau g) x, x-x^{*}\right\rangle \\
& =\left\langle(I+\tau g-D) x-x, x^{*}-x\right\rangle \\
& \leq 0 .
\end{aligned}
$$

Also, we show the uniqueness of a solution of the variational inequality

$$
\left\langle(D-\tau g) x, x-x^{*}\right\rangle \leq 0, \quad x^{*} \in \Gamma .
$$

Suppose that $x \in \Gamma$ and $x^{*} \in \Gamma$ are both solutions of 3.26 ; then

$$
\left\langle(D-\tau g) x, x-x^{*}\right\rangle \leq 0
$$

and

$$
\left\langle(D-\tau g) x^{*}, x^{*}-x\right\rangle \leq 0 .
$$

Adding the last two inequalities we have

$$
\left\langle(D-\tau g) x-(D-\tau g) x^{*}, x-x^{*}\right\rangle \leq 0 .
$$

Since $D-\tau g$ is strongly monotone by Lemma 2.6, we have that $x=x^{*}$. Hence the uniqueness is proved.

Lastly, we prove that $x_{n} \rightarrow x^{*}$ as $n \rightarrow \infty$. From (3.1) and (3.7) we obtain

$$
\begin{aligned}
\left\|x_{n+1}-x^{*}\right\|^{2}= & \left\langle\beta_{n} \tau g\left(x_{n}\right)+\left(I-\beta_{n} D\right) y_{n}-x^{*}, x_{n+1}-x^{*}\right\rangle \\
= & \beta_{n}\left\langle\tau g\left(x_{n}\right)-D x^{*}, x_{n+1}-x^{*}\right\rangle+\left\langle\left(I-\beta_{n} D\right)\left(y_{n}-x^{*}\right), x_{n+1}-x^{*}\right\rangle \\
\leq & \beta_{n} \tau\left\langle g\left(x_{n}\right)-g\left(x^{*}\right), x_{n+1}-x^{*}\right\rangle+\beta_{n}\left\langle\tau g\left(x^{*}\right)-D x^{*}, x_{n+1}-x^{*}\right\rangle \\
& +\left(1-\beta_{n} \bar{\tau}\right)\left\|y_{n}-x^{*}\right\|\left\|x_{n+1}-x^{*}\right\| \\
\leq & \beta_{n} \tau \mu\left\|x_{n}-x^{*}\right\|\left\|x_{n+1}-x^{*}\right\|+\beta_{n}\left\langle\tau g\left(x^{*}\right)-D x^{*}, x_{n+1}-x^{*}\right\rangle \\
& +\left(1-\beta_{n} \bar{\tau}\right)\left\|x_{n}-x^{*}\right\|\left\|x_{n+1}-x^{*}\right\| \\
= & {\left[1-(\bar{\tau}-\tau \mu) \beta_{n}\right]\left\|x_{n}-x^{*}\right\|\left\|x_{n+1}-x^{*}\right\| } \\
& +\beta_{n}\left\langle\tau g\left(x^{*}\right)-D x^{*}, x_{n+1}-x^{*}\right\rangle \\
\leq & \frac{1-(\bar{\tau}-\tau \mu) \beta_{n}}{2}\left(\left\|x_{n}-x^{*}\right\|^{2}+\left\|x_{n+1}-x^{*}\right\|^{2}\right) \\
& +\beta_{n}\left\langle\tau g\left(x^{*}\right)-D x^{*}, x_{n+1}-x^{*}\right\rangle \\
\leq & \frac{1-(\bar{\tau}-\tau \mu) \beta_{n}}{2}\left\|x_{n}-x^{*}\right\|^{2}+\frac{1}{2}\left\|x_{n+1}-x^{*}\right\|^{2} \\
& +\beta_{n}\left\langle\tau g\left(x^{*}\right)-D x^{*}, x_{n+1}-x^{*}\right\rangle .
\end{aligned}
$$

Then, it follows that

$\left\|x_{n+1}-x^{*}\right\|^{2} \leq\left[1-(\bar{\tau}-\tau \mu) \beta_{n}\right]\left\|x_{n}-x^{*}\right\|^{2}+\beta_{n}(\bar{\tau}-\tau \mu) \frac{2\left\langle\tau g\left(x^{*}\right)-D x^{*}, x_{n+1}-x^{*}\right\rangle}{(\bar{\tau}-\tau \mu)}$.

By conditions (i) and (ii) of Lemma 3.2 , we obtain $\lim _{n \rightarrow \infty}\left\|x_{n}-x^{*}\right\|=0$ using Lemma 2.7 
Case 2: Assume that $\left\{\left\|x_{n}-p\right\|\right\}$ is not a monotonically increasing sequence. Set $\Theta_{n}=\left\|x_{n}-p\right\|^{2}$ and let $\sigma: \mathbb{N} \rightarrow \mathbb{N}$ be a mapping for all $n \geq n_{0}$ (for some $n_{0}$ large enough) defined by

$$
\sigma(n):=\max \left\{k \in \mathbb{N}: k \leq n, \Theta_{k} \leq n, \Theta_{k} \leq \Theta_{k+1}\right\} .
$$

Clearly, $\sigma$ is a non-decreasing sequence such that $\sigma(n) \rightarrow 0$ as $n \rightarrow \infty$ and $\Theta_{\sigma(n)} \leq$ $\Theta_{\sigma(n)+1}$, for $n \geq n_{0}$. It follows from 3.22 that

$$
\begin{aligned}
\alpha_{\sigma(n)}\left(1-\alpha_{\sigma(n)}\right)(1- & \left.\beta_{\sigma(n)} \bar{\tau}\right)^{2}\left\|K u_{\sigma(n)}-u_{\sigma(n)}\right\|^{2} \\
\leq & \left(1-\beta_{\tau(n)} \bar{\tau}\right)^{2}\left\|x_{\sigma(n)}-p\right\|^{2}-\left\|x_{\sigma(n)+1}-p\right\|^{2} \\
& +\beta_{\sigma(n)}\left\|\tau g\left(x_{\sigma(n)}\right)-D p\right\|^{2} \\
& +2 \beta_{\sigma(n)}\left(1-\beta_{\sigma(n)} \bar{\tau}\right)\left\|\tau g\left(x_{\sigma(n)}\right)-D p\right\|\left\|y_{\sigma(n)}-p\right\| .
\end{aligned}
$$

Therefore, since $\lim _{n \rightarrow \infty} \beta_{\sigma(n)}=0$, we obtain

$$
\lim _{n \rightarrow \infty}\left\|K u_{\sigma(n)}-u_{\sigma}\right\|=0 .
$$

Following the same argument as in Case 1 , we conclude that $\left\{x_{\sigma}\right\},\left\{y_{\sigma}\right\}$, and $\left\{u_{\sigma}\right\}$ converge weakly to $p \in F(K) \cap \Omega$. Now, for all $n \geq n_{0}$, we have

$$
\begin{aligned}
0 \leq & \left\|x_{\sigma(n)+1}-x^{*}\right\|^{2}-\left\|x_{\sigma(n)}-x^{*}\right\|^{2} \\
\leq & \left(1-\beta_{\sigma(n)} \bar{\tau}\right)\left\|x_{\sigma(n)}-x^{*}\right\|^{2}+\beta_{\sigma(n)}^{2}\left\|\tau g\left(x_{\sigma}\right)-D x^{*}\right\|^{2} \\
& +2 \beta_{\sigma(n)}\left(1-\beta_{\sigma(n)}\right) \bar{\sigma}\left\|\tau g\left(x_{\sigma(n)}\right)-D x^{*}\right\|\left\|x_{\sigma(n)}-x^{*}\right\|-\left\|x_{\sigma(n)}-x^{*}\right\|^{2} \\
= & -\beta_{\sigma(n)} \bar{\sigma}\left\|x_{\tau(n)}-x^{*}\right\|^{2}+\beta_{\sigma(n)}^{2}\left\|\tau g\left(x_{\sigma(n)}\right)-D x^{*}\right\|^{2} \\
& +2 \beta_{\sigma(n)}\left(1-\beta_{\sigma(n)} \bar{\sigma}\right)\left\langle\tau g\left(x_{\tau(n)}\right)-D x^{*}, x_{\sigma(n)+1}-x^{*}\right\rangle .
\end{aligned}
$$

Thus,

$$
\begin{aligned}
\left\|x_{\sigma(n)}-x^{*}\right\|^{2} \leq & \frac{\beta_{\sigma(n)}}{\bar{\sigma}}\left\|\tau g\left(x_{\sigma(n)}\right)-D x^{*}\right\|^{2} \\
& +\frac{2\left(1-\beta_{\sigma(n)} \bar{\sigma}\right)}{\bar{\sigma}}\left\langle\tau g\left(x_{\sigma(n)}\right)-D x^{*}, x_{\sigma(n)+1}-x^{*}\right\rangle .
\end{aligned}
$$

Since $\beta_{\sigma(n)} \rightarrow 0$ as $n \rightarrow \infty$ and $\limsup _{n \rightarrow \infty}\left\langle\tau g\left(x_{\sigma(n)}\right)-D x^{*}, x_{\sigma(n)+1}-x^{*}\right\rangle \leq 0$, we conclude that $\left\{x_{\sigma}\right\}$ converges to $x^{*}$.

Corollary 3.4. Let $C$ and $Q$ be nonempty closed convex subsets of real Hilbert spaces $H_{1}$ and $H_{2}$ respectively. Let $A: H_{1} \rightarrow H_{2}$ be a bounded linear operator and $T: H_{1} \rightarrow H_{2}$ be a nonexpansive mapping. Let $F_{1}: C \times C \rightarrow \mathbb{R}, F_{2}: Q \times Q \rightarrow \mathbb{R}$ be bifunctions satisfying (A1)-(A4), and let $D$ be a strongly positive bounded linear operator on $H_{1}$ with coefficient $\bar{\tau}>0$. Let $f_{1}: H_{1} \rightarrow H_{1}$ be a $\rho_{1}$-inverse strongly monotone mapping and $f_{2}: H_{2} \rightarrow H_{2}$ be a $\rho_{2}$-inverse strongly monotone mapping. Assume that $\Gamma:=F(T) \cap \Omega \neq \emptyset$ and that $g$ is a contraction mapping 
with coefficient $\mu \in(0,1)$. Let the sequences $\left\{u_{n}\right\},\left\{y_{n}\right\}$, and $\left\{x_{n}\right\}$ be generated for arbitrary $x_{1} \in H$ by

$$
\left\{\begin{aligned}
u_{n} & =T_{r_{n}}^{F_{1}}\left(I-r_{n} f_{1}\right)\left(x_{n}+\gamma_{n} A^{*}\left(T_{s_{n}}^{F_{2}}\left(I-s_{n} f_{2}\right)-I\right) A x_{n}\right), \\
y_{n} & =\alpha_{n} u_{n}+\left(1-\alpha_{n}\right) T u_{n}, \\
x_{n+1} & =\beta_{n} \tau g\left(x_{n}\right)+\left(I-\beta_{n} D\right) y_{n}, n \geq 1,
\end{aligned}\right.
$$

where $0<r \leq r_{n}<2 \rho_{1}, 0<s \leq s_{n}<2 \rho_{2}, \alpha_{n} \in(0,1)$, and the step size $\gamma_{n}$ is chosen in such a way that for some $\epsilon>0$,

$$
\gamma_{n} \in\left(\epsilon, \frac{\left\|\left(T_{s_{n}}^{F_{2}}\left(I-s_{n} f_{2}\right)-I\right) A x_{n}\right\|^{2}}{\left\|A^{*}\left(T_{s_{n}}^{F_{2}}\left(I-s_{n} f_{2}\right)-I\right) A x_{n}\right\|^{2}}-\epsilon\right),
$$

for all $T_{s_{n}}^{F_{2}}\left(I-s_{n} f_{2}\right) A x_{n} \neq A x_{n}$, and $\gamma_{n}=\gamma$ otherwise $(\gamma$ being any nonnegative real number), with the sequence $\left\{\beta_{n}\right\},\left\{\xi_{n}\right\},\left\{\eta_{n}\right\}$ satisfying the following conditions:

(i) $\beta_{n} \in(0,1), \lim _{n \rightarrow \infty} \beta_{n}=0$, and $\sum_{n=1}^{\infty} \beta_{n}=\infty$;

(ii) $0<\tau<\frac{\bar{\tau}}{\mu}$;

(iii) $0<\liminf _{n \rightarrow \infty} \alpha_{n} \leq \limsup _{n \rightarrow \infty} \alpha_{n}<1$.

Then, the sequence $\left\{x_{n}\right\}$ generated by (3.1) converges strongly to $x^{*} \in \Gamma$ which solves the variational inequality

$$
\left\langle(D-\tau g) x^{*}, x^{*}-x\right\rangle \leq 0, \quad \forall x \in \Gamma .
$$

\section{A numerical EXAmple}

We consider a numerical example of our algorithm in $\mathbb{R}^{2}$.

Let $H_{1}=H_{2}=\mathbb{R}^{2}$. Then for $z=\left(z_{1}, z_{2}\right), y=\left(y_{1}, y_{2}\right),\left(u_{1}, u_{2}\right),\left(v_{1}, v_{2}\right)$, and $\left(x_{1}, x_{2}\right)$, define $F_{i}(z, y)=-3 i z^{2}+2 i y z+4 i y^{2}, i=1,2$. Then, Lemma 2.3 ensures that we can find $x \in \mathbb{R}^{2}$ such that

$$
\begin{aligned}
& F_{i}(z, y)+\frac{1}{r_{n}}\langle y-z, z-x\rangle \geq 0, \quad \forall y \in \mathbb{R}^{2}, i=1,2 \\
& \Longleftrightarrow-3 i z^{2}+2 i y z+4 i y^{2}+\frac{1}{r_{n}}\left(y z-x y-z^{2}+x z\right) \geq 0 \\
& \Longleftrightarrow-3 i r_{n} z^{2}+2 i r_{n} y z+4 i r_{n} y^{2}+y z-x y-z^{2}+z x .
\end{aligned}
$$

Let $P(y)=4 i r_{n} y^{2}+\left(2 i r_{n} z+z-x\right) y-3 i r_{n} z^{2}-z^{2}+z x$. Then $P$ is a quadratic equation in $y$. Thus, we obtain the determinant as follows:

$$
\begin{aligned}
\Delta & =b^{2}-4 a c \\
& =\left(2 i r_{n} z+z-x\right)^{2}-4 i r_{n}\left(-3 i r_{n} z^{2}-z^{2}+z x\right) \\
& =16 i^{2} r_{n}^{2} z^{2}+8 i r_{n} z^{2}-8 i r_{n} x z-2 x z+z^{2}+x^{2} \\
& =x^{2}-2\left(4 i r_{n} z+z\right) x+\left(4 i r_{n} z+z\right)^{2} \\
& =\left(x-\left(4 i r_{n}+1\right) z\right)^{2} \\
& \geq 0 .
\end{aligned}
$$


Since $P(y) \geq 0$ for all $y \in \mathbb{R}$, it has at most one solution in $\mathbb{R}$, and then $\Delta \leq 0$. So we have that $z=\frac{x}{4 i r_{n}+1}$. Hence,

$$
T_{r_{n}}^{F_{1}}(x)=\left(\frac{x_{1}}{4 i r_{n}+1}, \frac{x_{2}}{4 i r_{n}+1}\right), \quad i=1,2 .
$$

Let $f_{i}=\frac{x}{2 i}, i=1,2$. Then $f_{i}$ is $\rho_{i}$-inverse strongly monotone with $\rho_{i}=2 i, i=1,2$. Let $A(x)=\left(4 x_{1}+3 x_{2}, 3 x_{1}+2 x_{2}\right)$ and $D(x)=2 x$ and $g(x)=\frac{x}{3}$. Then, $\bar{\tau}=2$ and $\mu=\frac{1}{3}$. Thus, we can take $\tau=2$ and condition (iii) of Theorem 3.3 is satisfied. From (4.1) we get that $T_{r_{n}}^{F_{1}}(x)=\left(\frac{x_{1}}{4 r_{n}+1}, \frac{x_{2}}{4 r_{n}+1}\right)$ and $T_{s_{n}}^{F_{2}}(x)=\left(\frac{x_{1}}{8 r_{n}+1}, \frac{x_{2}}{8 r_{n}+1}\right)$. Thus, we take $r_{n}=\frac{3 n}{2 n+1}$ and $s_{n}=\frac{7 n-1}{3 n+4}$ for all $n \geq 1$.

Now we define $T: \mathbb{R}^{2} \rightarrow \mathbb{R}^{2}$ by

$$
T\left(x_{1}, x_{2}\right)=-\left(\frac{2 \alpha+1}{2}\right)\left(x_{1}, x_{2}\right), \quad \forall \alpha>\frac{1}{2} .
$$

Then $T$ is an $L$-Lipschitzian and quasi-pseudocontractive mapping, with $L=$ $\left(\frac{2 \alpha+1}{2}\right)^{2}>1, \forall \alpha>\frac{1}{2}$. We now take $\beta_{n}=\frac{1}{n+2}, \alpha_{n}=\frac{n}{2 n+3}, \eta_{n}=\frac{1}{2+\left(\frac{2 \alpha+1}{2}\right)^{4}}$, and $\xi_{n}=\frac{8}{(2 \alpha+1)^{4}} \forall n \geq 1, \alpha>\frac{1}{2}$. Then conditions (i) and (ii) of Lemma 3.2 are satisfied. Hence, for $x_{1} \in \mathbb{R}^{2}$, Algorithm 3.1 becomes

$$
\left\{\begin{aligned}
u_{n} & =T_{r_{n}}^{F_{1}}\left(I-r_{n} f_{1}\right)\left(x_{n}+\gamma_{n} A^{*}\left(T_{s_{n}}^{F_{2}}\left(I-s_{n} f_{2}\right)-I\right) A x_{n}\right), \\
y_{n} & \left.=\frac{n}{2 n+3} u_{n}+\frac{n+3}{2 n+3}\left(\left(1-\xi_{n}\right) I+\xi_{n} T\left(\left(1-\eta_{n}\right)\right) I+\eta_{n} T\right)\right) u_{n} \\
x_{n+1} & =\frac{2}{3(n+2)} x_{n}+\left(1-\frac{2}{n+1}\right) y_{n} .
\end{aligned}\right.
$$

These three cases are displayed in Figure 1 on the next page:

Case 1: $x_{0}=(0.1,0.5)^{T}$ and $\alpha=2$.

Case 2: $x_{0}=(0.1,0.5)^{T}$ and $\alpha=1$.

Case 3: $x_{0}=(1,-2)^{T}$ and $\alpha=2$. 

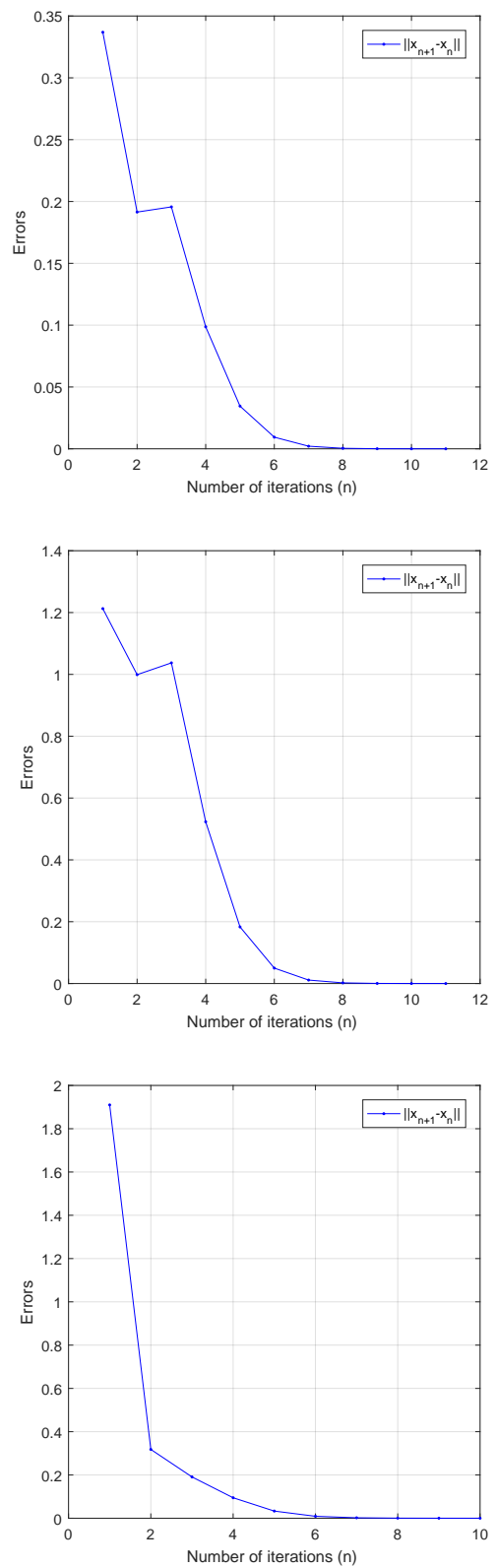

Figure 1. Errors vs Number of iterations $(n)$ : Case 1 (top); Case 2 (middle); Case 3 (bottom). 


\section{ACKNowledGment}

The authors would like to thank the anonymous referee for useful comments which led to a great improvement of this paper.

\section{REFERENCES}

[1] H. A. Abass, F. U. Ogbuisi and O. T. Mewomo, Common solution of split equilibrium problem and fixed point problem with no prior knowledge of operator norm, Politehn. Univ. Bucharest Sci. Bull. Ser. A Appl. Math. Phys. 80 (2018), no. 1, 175-190. MR 3785191.

[2] H. A. Abass, C. C. Okeke and O. T. Mewomo, On split equality mixed equilibrium and fixed point problems for countable families of generalized $K_{1}$-strictly pseudo-contractive multivalued mappings, Dyn. Contin. Discrete Impuls. Syst. Ser. B Appl. Algorithms 25 (2018), no. 6, 369-395. MR 3925176 .

[3] H. A. Abass, A. A. Mebawondu and O. T. Mewomo, Some results for a new three steps iteration scheme in Banach spaces, Bull. Transilv. Univ. Braşov Ser. III 11(60) (2018), no. 2, 1-18. MR 3909125.

[4] E. Blum and W. Oettli, From optimization and variational inequalities to equilibrium problems, Math. Student 63 (1994), no. 1-4, 123-145. MR 1292380.

[5] C. Byrne, Iterative oblique projection onto convex sets and the split feasibility problem, Inverse Problems 18 (2002), no. 2, 441-453. MR 1910248

[6] G. Cai, Y. Shehu and O. S. Iyiola, Viscosity iterative algorithms for fixed point problems of asymptotically nonexpansive mappings in the intermediate sense and variational inequality problems in Banach spaces, Numer. Algorithms 76 (2017), no. 2, 521-553. MR 3704880

[7] Y. Censor and T. Elfving, A multiprojection algorithm using Bregman projections in a product space, Numer. Algorithms 8 (1994), no. 2-4, 221-239. MR 1309222

[8] Y. Censor et al., The multiple-sets split feasibility problem and its applications for inverse problems, Inverse Problems 21 (2005), no. 6, 2071-2084. MR 2183668

[9] Y. Censor, T. Bortfeld, B. Martin and A. Trofimov, A unified approach for inversion problems in intensity-modulated radiation therapy, Phys. Med. Biol. 51 (2006), 2353-2365. https://doi.org/10.1088/0031-9155/51/10/001

[10] Y. Censor, A. Gibali and S. Reich, Algorithms for the split variational inequality problem, Numer. Algorithms 59 (2012), no. 2, 301-323. MR 2873136

[11] S. Chang, H. W. Joseph Lee and C. K. Chan, A new method for solving equilibrium problem fixed point problem and variational inequality problem with application to optimization, Nonlinear Anal. 70 (2009), no. 9, 3307-3319. MR 2503077

[12] S. Chang, L. Wang and L.-J. Qin, Split equality fixed point problem for quasi-pseudocontractive mappings with applications, Fixed Point Theory Appl. 2015, 2015:208, 12 pp. MR 3424795

[13] K. Cheawchan and A. Kangtunyakarn, The modified split generalized equilibrium problem for quasi-nonexpansive mappings and applications, J. Inequal. Appl. 2018, Paper No. 122, 28 pp. MR 3805674

[14] P. L. Combettes, The convex feasibility problem in image recovery, Adv. Imaging Electron Phys. 95 (1996), 155-453. https://doi.org/10.1016/S1076-5670(08)70157-5

[15] P. L. Combettes and S. A. Hirstoaga, Equilibrium programming in Hilbert spaces, J. Nonlinear Convex Anal. 6 (2005), no. 1, 117-136. MR 2138105

[16] J. Deepho et al., A new general iterative scheme for split variational inclusion and fixed point problems of $k$-strict pseudo-contraction mappings with convergence analysis, J. Comput. Appl. Math. 318 (2017), 293-306. MR 3602701

[17] L. O. Jolaoso et al., A self adaptive inertial subgradient extragradient algorithm for variational inequality and common fixed point of multivalued mappings in Hilbert spaces, Demonstr. Math. 52 (2019), no. 1, 183-203. MR 3938331 
[18] K. R. Kazmi and S. H. Rizvi, Iterative approximation of a common solution of a split equilibrium problem, a variational inequality problem and a fixed point problem, J. Egyptian Math. Soc. 21 (2013), no. 1, 44-51. MR 3040758

[19] G. Marino and H.-K. Xu, A general iterative method for nonexpansive mappings in Hilbert spaces, J. Math. Anal. Appl. 318 (2006), no. 1, 43-52. MR 2210870.

[20] B. Qu and N. Xiu, A note on the $C Q$ algorithm for the split feasibility problem, Inverse Problems 21 (2005), no. 5, 1655-1665. MR 2173415

[21] H. R. Sahebi and S. Ebrahimi, A viscosity iterative algorithm for the optimization problem system, Filomat 31 (2017), no. 8, 2249-2266. MR 3637024

[22] S. Suantai et al., On solving split equilibrium problems and fixed point problems of nonspreading multi-valued mappings in Hilbert spaces, Fixed Point Theory Appl. 2016, Paper No. 35, 16 pp. MR 3481192

[23] A. Taiwo, L. O. Jolaoso and O. T. Mewomo, A modified Halpern algorithm for approximating a common solution of split equality convex minimization problem and fixed point problem in uniformly convex Banach spaces, Comput. Appl. Math. 38 (2019), no. 2, Paper No. 77, 28 pp. MR 3933584

[24] A. Taiwo, L. O. Jolaoso and O. T. Mewomo, Parallel hybrid algorithm for solving pseudomonotone equilibrium and split common fixed point problems, Bull. Malays. Math. Sci. Soc. 43 (2020), no. 2, 1893-1918. MR 4061458

[25] W. Takahashi and M. Toyoda, Weak convergence theorems for nonexpansive mappings and monotone mappings, J. Optim. Theory Appl. 118 (2003), no. 2, 417-428. MR 2006529

[26] H.-K. Xu, Iterative methods for the split feasibility problem in infinite-dimensional Hilbert spaces, Inverse Problems 26 (2010), no. 10, 105018, 17 pp. MR 2719779

[27] H.-K. Xu, Iterative algorithms for nonlinear operators, J. London Math. Soc. (2) 66 (2002), no. 1, 240-256. MR 1911872

[28] J. Zhao and S. Wang, Viscosity approximation methods for the split equality common fixed point problem of quasi-nonexpansive operators, Acta Math. Sci. Ser. B (Engl. Ed.) 36 (2016), no. 5, 1474-1486. MR 3529631

Hammed Anuoluwapo Abass

School of Mathematics, Statistics and Computer Science, University of KwaZulu-Natal, Durban, South Africa

216075727@stu.ukzn.ac.za

Chinedu Izuchukwu

School of Mathematics, Statistics and Computer Science, University of KwaZulu-Natal, Durban, South Africa

izuchukwuc@ukzn.ac.za

Oluwatosin Temitope Mewomo ${ }^{\otimes}$

School of Mathematics, Statistics and Computer Science, University of KwaZulu-Natal, Durban, South Africa

mewomoo@ukzn.ac.za

Received: October 27, 2018

Accepted: August 6, 2019 